\title{
Mapa estratégico para programas de recursos humanos: avaliando o desempenho de Redes Bayesianas
}

\section{Strategic map to programs of human resources: evaluating the performance of Bayesian Networks}

\author{
Ricardo José Ferreira ${ }^{1}$ \\ Paulo Estevão Lemos de Oliveira ${ }^{1}$ \\ Paulo Renato Alves Firmino² \\ Enrique Andrés López Droguett ${ }^{1}$
}

\begin{abstract}
Resumo: Visando desenvolver mão de obra especializada na área de petróleo, gás natural e biocombustíveis, a Agência Nacional do Petróleo - ANP - tem promovido programas de capacitação de Recursos Humanos (PRH) em todo o País. Com o objetivo de elaborar as estratégias subjacentes a tais programas de maneira estruturada e abrangente, métodos de administração tais como Balanced Scorecard (BSC) vêm sendo adotados. Contudo, infortúnios como a não mensuração das incertezas envolvidas até o alcance dos objetivos almejados sugerem a adoção de formalismos mais sofisticados, tais como redes Bayesianas (RB), em detrimento da BSC. Em se tratando de RBs, o desafio passa a ser uma quantificação adequada dos seus parâmetros, possibilitando estimativas confiáveis a partir delas. O objetivo do presente trabalho é avaliar o desempenho de RBs no suporte a um dado problema real de $\mathrm{PRH}$, a partir do confronto entre seus prognósticos e o que foi de fato observado. O modelo em questão foi elaborado a partir de um BSC e posteriormente quantificado de acordo com opiniões de especialistas, devido à total ausência de dados empíricos relevantes. A RB foi delineada para dar suporte à definição e implantação de estratégias para o desenvolvimento científico e abertura de mercados especializados para dado curso de engenharia de uma universidade federal do País. As análises realizadas indicam o bom desempenho do modelo e apontam como um dos principais componentes responsáveis o método de quantificação adotado.
\end{abstract}

Palavras-chave: Programa de recursos humanos. Planejamento estratégico. Balanced scorecard. Redes Bayesianas. Métodos de edução. Coleta de opiniões.

\begin{abstract}
In order to develop manpower expertise in the area of oil, natural gas and biofuels, the National Petroleum Agency - NPA - has been promoting training programs for Human Resources (PHR). Aimed at determining the strategies behind these programs in a structured and comprehensive manner, methods of administration such as Balanced Scorecard (BSC) have been adopted. However, failures such as non-measurement of uncertainties involved up until the desired goals have been reached, suggest the adoption of more sophisticated formulae, such as Bayesian networks $(B N)$ rather than BSC. In the case of $B N$, the challenge becomes a proper quantification of its parameters, allowing reliable estimates from them. The objective of this work is to evaluate the performance of $B N$ in handling a given real PHR problem by means of the comparison between its predictions and the respective observed results. The model has been developed from a BSC and subsequently quantified according to expert opinions, since no relevant empirical data was available. The BN was designed to support the deployment of strategies for scientific development and opening of markets for a given university course. The analyses carried out indicate the good performance of the model, emphasizing the adopted elicitation method in such a process.
\end{abstract}

Keywords: Program for human resources. Strategic planning. Balanced scorecard. Bayesian Networks. Methods of elicitation. Collection of opinions.

\section{Introdução}

Para desenvolver pesquisas sobre recursos humanos, a Agência Nacional do Petróleo -ANP - vem criando

programas em vários estados do Brasil com o objetivo de obter melhores formas de gerir os recursos

\footnotetext{
${ }^{1}$ Universidade Federal de Pernambuco - UFPE, Av. Prof. Moraes Rego, 1235, Cidade Universitária, CEP 50670-901, Recife - PE, Brasil, E-mails: rickhardo@gmail.com; paestevao@hotmail.com; ealopez@ufpe.br

${ }^{2}$ Universidade Federal Rural de Pernambuco - UFRPE, Rua Dom Manoel de Medeiros, s/n, Dois Irmãos, CEP 52171-900, Recife - PE, Brasil, E-mails: paulo.firmino@deinfo.ufrpe.br
}

Recebido em 23/7/2009 — Aceito em 17/2/2010

Suporte financeiro: Programa de Recursos Humanos da ANP. 
humanos sobre as fontes derivadas do petróleo e gás industrial. Estes programas são denominados de Programas de Recursos Humanos - PRH - e atingem quase todo o País, envolvendo atualmente 36 projetos em 16 estados. O objetivo geral dos PRHs é recrutar profissionais e treiná-los para funções correlatas às áreas de petróleo, gás natural e biocombustíveis. Especificamente, os objetivos do PRH em questão são de formação profissional para projetar, operar e reparar unidades de processamento de gás. A ANP adota uma política para designar verba aos PRHs da seguinte forma: quanto melhor os resultados de um PRH, maior será a verba a ele destinada. Os resultados considerados positivos são desde pesquisa realizada a partir da graduação até patrocínios provenientes de empresas externas, levando à criação de Universidades-Empresas, por exemplo. Durante os anos de 2006 e 2007, o desempenho do PRH sob estudo no presente trabalho estava entre os dez últimos dentre os 36 mantidos pela ANP. Isto reflete a ineficácia e ineficiência da política administrativa vivida pelo programa nesse período para alcançar as metas exigidas pela ANP. Com o objetivo de contornar este problema, Oliveira (2007) propõe um passo inicial no sentido da administração estratégica, a partir de pontos como a missão do PRH, sua visão, seu mapa estratégico associado e assim por diante. Pode-se observar que, como várias organizações em torno do mundo, o PRH sofre de falhas administrativas, embora esteja naturalmente em busca dos melhores resultados.

Oliveira (2007) aponta para a possibilidade de modelagem do problema de PRH por meio da metodologia administrativa de Balanced Scorecard (BSC). Inicialmente proposto por Kaplan e Norton (1997), BSC tem por principal objetivo relacionar as diferentes perspectivas - Financeira, Mercado, Processos Internos e Aprendizado e Crescimento - de uma organização de maneira estruturada e intuitiva para os membros componentes de dado sistema de produção, desde a alta gerência até os funcionários do mais baixo escalão. Contudo, algumas deficiências de BSC reduzem sua capacidade de realizar prognósticos, característica fundamental quando o tema sob estudo é estratégia. BSC não apresenta um formalismo de modelagem da incerteza, inviabilizando assim uma análise de riscos. Além de descriminar as relações entre as diferentes perspectivas de maneira determinística (se dada sequência de tarefas delineada pelo BSC for adequadamente realizada então se alcança, certamente, a meta pré-estabelecida segundo o modelo), BSC não quantifica as probabilidades associadas aos eventos de interesse. A quantificação realizada pelo BSC envolve metas e indicadores de desempenho os quais servem de referência para o alcance dos objetivos. Recomenda-se Norreklit (2000) para maiores detalhes. Além disso, Akkermans e Oorschot (2005) relatam que, mesmo com a utilização de BSC para a modelagem da estratégia, não se tem certeza se todas as relações de causa e efeito estão devidamente determinadas, enquanto que autores como Othman (2006) apontam para objetivos que poderiam ser excluídos e outros, incluídos, após um processo de testes preliminares da estratégia realizado com os gestores por um determinado tempo.

Logo, além da devida determinação das relações existentes e dos objetivos relevantes, a mensuração dos riscos decorre a partir da quantificação das incertezas inerentes ao problema. Trabalhar com a quantificação dos riscos que cercam os objetivos e relações se torna importante para o conhecimento dos gestores. Neste sentido, Oliveira (2007) propõe a introdução de redes Bayesianas como formalismo que generaliza BSC, possibilitando dentre outras coisas, lidar com o problema da escassez de dados com a introdução de opiniões de especialistas, por sua vez quantificadas a partir de métodos de edução e coleta de julgamentos. Devido à total escassez de dados quando da abordagem do seu problema de PRH, Oliveira (2007) recorre a um método de edução que se utiliza de algoritmos de busca computacionais e intervalos de crença Bayesianos. Assim como os demais métodos de edução do conhecimento classificados como estruturados, o método adotado busca explorar a opinião do especialista de maneira que ele seja o mais fiel possível ao apresentar suas próprias incertezas. O método se utiliza do algoritmo de busca binária de forma a dividir um determinado intervalo em dois subintervalos cada qual com a devida credibilidade fornecida pelo especialista. Dentre as apresentações deste método, recomenda-se Lins et al. (2007) e Firmino et al. (2008).

Usando o modelo apresentado por Oliveira (2007), Ferreira et al. (2008) apresentam várias possíveis análises feitas pela simulação da RB montada a partir do BSC do caso PRH em questão. Em seu trabalho, eles mostram uma aplicação direta do modelo proposto por Oliveira (2007), dando especial ênfase a uma das quatro perspectivas do BSC-RB resultante. Análises de prognóstico foram enfatizadas em uma fase preliminar, quando o BSC ainda não tinha sido implantado.

Com isso, mesmo antes de executar a estratégia, os especialistas podem remodelar os objetivos de maneira a torná-los cada vez mais possíveis de serem alcançados. É importante ainda notar que, inicialmente, uma RB que corresponda a determinado BSC tende a modelar as mesmas relações de causa e efeito apresentadas pelos gestores. Mas devido à capacidade de prognóstico presente na RB, novas relações de causa e efeito podem ser introduzidas enquanto que as existentes, remodeladas se necessário, o que preenche a deficiência de prognóstico presente no BSC. 
As análises de prognóstico foram fundamentais para a manutenção do BSC do PRH antes mesmo da sua execução. Durante o ano de 2008, o BSC do PRH sob estudo foi implantado de acordo com a análise fornecida pelo modelo BSC-BBN. Visando criar um feedback aos gestores do programa ao mesmo tempo em que se mede o desempenho do modelo em relação aos resultados, uma comparação entre os resultados esperados com os resultados observados torna tal feedback possível. A partir da coleta dos resultados observados com os gestores do PRH, esse trabalho realiza uma análise de desempenho ao comparar tais resultados com os previstos anteriormente, por análises de diagnósticos e análises retroativas (backward propagation).

Na seção 2, será apresentada uma breve análise sobre a história do PRH e o modelo proposto e suas previsões antes da realização da estratégia montada para o PRH. A seção 3 mostra os principais resultados da estratégia do PRH em 2008 em comparação com os resultados previstos. A seção 4 traz uma discussão sobre os resultados antes e depois da aplicação da estratégia.

\section{Programas de recursos humanos e sua modelagem via Redes Bayesianas}

O PRH estudado faz parte de uma rede de diversos projetos elaborados e distribuídos em 16 estados do Brasil pela ANP. Seu objetivo é o treinamento de profissionais nas áreas de petróleo e gás, especificamente na projeção, operação e manutenção do processo de gás e de unidades de refino relacionadas com o petróleo.

Anterior à explanação de cada passo da metodologia desenvolvida neste trabalho, um fluxograma de como se dá o processo de análise do PRH em questão vem a esclarecer como o problema é tratado pelo modelo proposto (Figura 1).
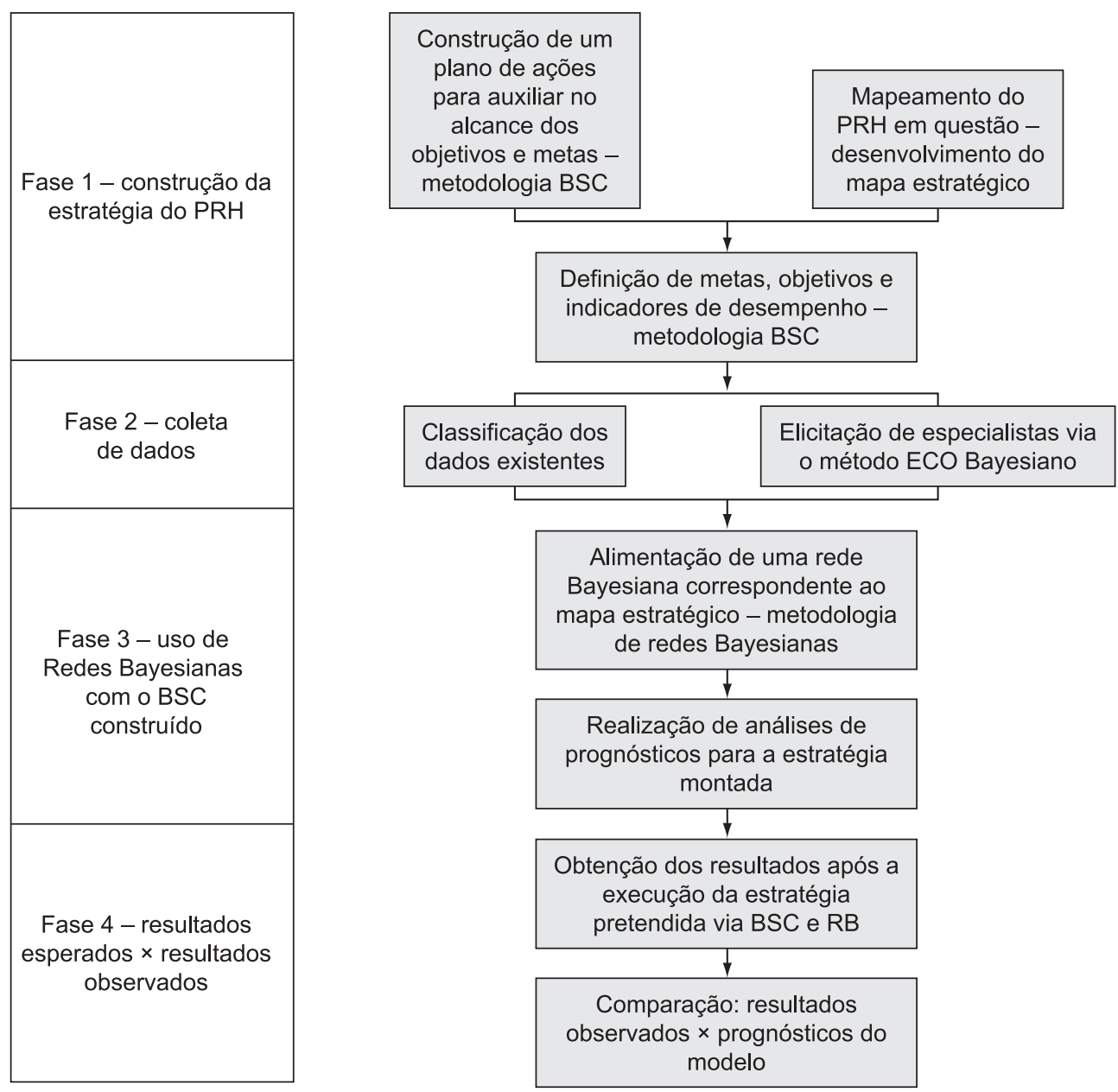

Figura 1. Fluxograma explanatório da metodologia desenvolvida para análise do problema em questão. 


\subsection{O uso do balanced scorecard na gestão do programa de recursos humanos}

A ANP criou uma maneira de avaliar o desempenho de PRH de acordo com uma série de requisitos contidos em um questionário enviado a todos os seus componentes. Dependendo de cada meta traçada pela ANP, dado PRH recebe uma pontuação e ganha uma classificação. Durante os anos de 2006 e 2007, o PRH em questão apareceu entre os últimos dez dentre todos no País. Isto é um sinal das suas limitações neste período. Com o objetivo de mudar essa realidade, os gestores do PRH refletiram sobre uma nova forma de administrá-lo.

Após uma busca por possíveis ferramentas gerenciais, os gestores do programa optaram pelo uso do BSC por se tratar de uma ferramenta com potencial de criar ligações entre diversos setores estratégicos dentro de uma organização, bem como afirmam seus criadores Kaplan e Norton (1997).

A principal característica do BSC é encontrada em sua estrutura, na qual se percebe uma visão da organização dividida em quatro blocos - denominados de perspectivas. Do ponto de vista da comunicação das estratégias dentro de uma organização, os quatro conjuntos de perspectivas (Perspectiva do Aprendizado e Crescimento, Perspectiva dos Processos Internos, Perspectivas do Mercado e Perspectiva do Cliente) estão interligados diretamente. Com todos os setores de posse da mesma informação estratégica, a realização da Perspectiva do Cliente ocorre como consequência de uma série de objetivos alcançados com sucesso em relação à estratégia da organização.

Tais objetivos são listados para cada conjunto e ilustrados a partir do Mapa Estratégico do BSC onde as perspectivas estão relacionadas por uma relação de causa e efeito (o sucesso ou fracasso de dados objetivos afeta no sucesso ou fracasso de outros). Ainda, cada objetivo possui um indicador para, de alguma forma, perceber o alcance ou não da meta traçada. Por último, um conjunto de ações é relacionado a cada objetivo, servindo como suporte de orientação para o cumprimento do respectivo objetivo.

Com a utilização do BSC, os gestores montaram um mapa estratégico para representar os principais objetivos a realizar, alinhando-se com os objetivos da ANP. Kaplan e Norton (2000) destacam a importância de ressaltar a organização dentro das organizações voltando os esforços sempre para a estratégia e, pensando nisso, um conjunto de ações foi elaborado para ajudar os gestores a alcançarem os objetivos. Primeiro, o mapa estratégico foi montado com os principais objetivos e indicadores de desempenho, como pode ser visto na Figura 2.
Além do mapa estratégico, um plano de ação foi montado para auxílio no alcance dos objetivos. A Tabela 1 mostra uma parte do plano de ações de alguns objetivos presentes no mapa estratégico delineado.

A Tabela 1 pode ser interpretada como um conjunto de ações programadas como auxílio no alcance dos objetivos. As colunas são autoexplicativas, à exceção das colunas "Resp", "Prior" e "Sit". A coluna "Resp" corresponde ao responsável por realizar aquela ação dentro da estratégia. A coluna "Prior" representa a prioridade que cada ação recebe indo de prioridades altas (preto) até prioridade básica (cinza). Por último, a coluna prazo nada mais representa senão o prazo estipulado para a realização de cada uma das ações programadas.

Mesmo com todas as ferramentas fornecidas pelo BSC, Blumenberg e Hinz (2006) comentam que ele não mede o quão provável é o alcance das metas na estratégia, enquanto que Herrero (2005) aborda o fato de que não se tem garantia que todas as relações de causa e efeito estão sendo modeladas. Baseado nessas deficiências, Oliveira (2007) propõe a abordagem de tais pontos por meio de um formalismo matemático conhecido como Redes Bayesianas (RB)

\subsection{Redes Bayesianas}

Korb e Nicholson (2003) definem redes Bayesianas como estruturas gráficas e de dados que representam razões ou argumentos no domínio da incerteza. Pearl (2000) data o início da década de 80 como a época de desenvolvimento das RBs, objetivando facilitar a tarefa de predição em sistemas de inteligência artificial para raciocínio com conhecimento incerto. As estruturas gráficas comentadas por Korb e Nicholson (2003) são, de fato, grafos acíclicos e direcionados em que os nós representam variáveis aleatórias e os arcos direcionados implicam em relações de causa e efeito entre as variáveis.

Sendo assim, tem-se que as RBs se mostram propícias a complementar as lacunas deixadas pelo BSC, principalmente no que tange à mensuração das incertezas inerentes à sua implementação, podendo simultaneamente modelar as relações de causa-efeito e quantificar probabilisticamente as chances de sucesso dos objetivos. Nesse contexto, especialistas são desafiados a inferir numericamente sobre suas incertezas acerca dos parâmetros da RB previamente montada diante da escassez de dados propriamente ditos. Tal escassez se mostrou como uma característica marcante do PRH abordado por Oliveira (2007) que, naquela oportunidade, recorreu a um método de edução e coleta de opiniões (ECO) de especialistas para que as incertezas acerca do parâmetro de interesse fossem adequadamente mensuradas. 


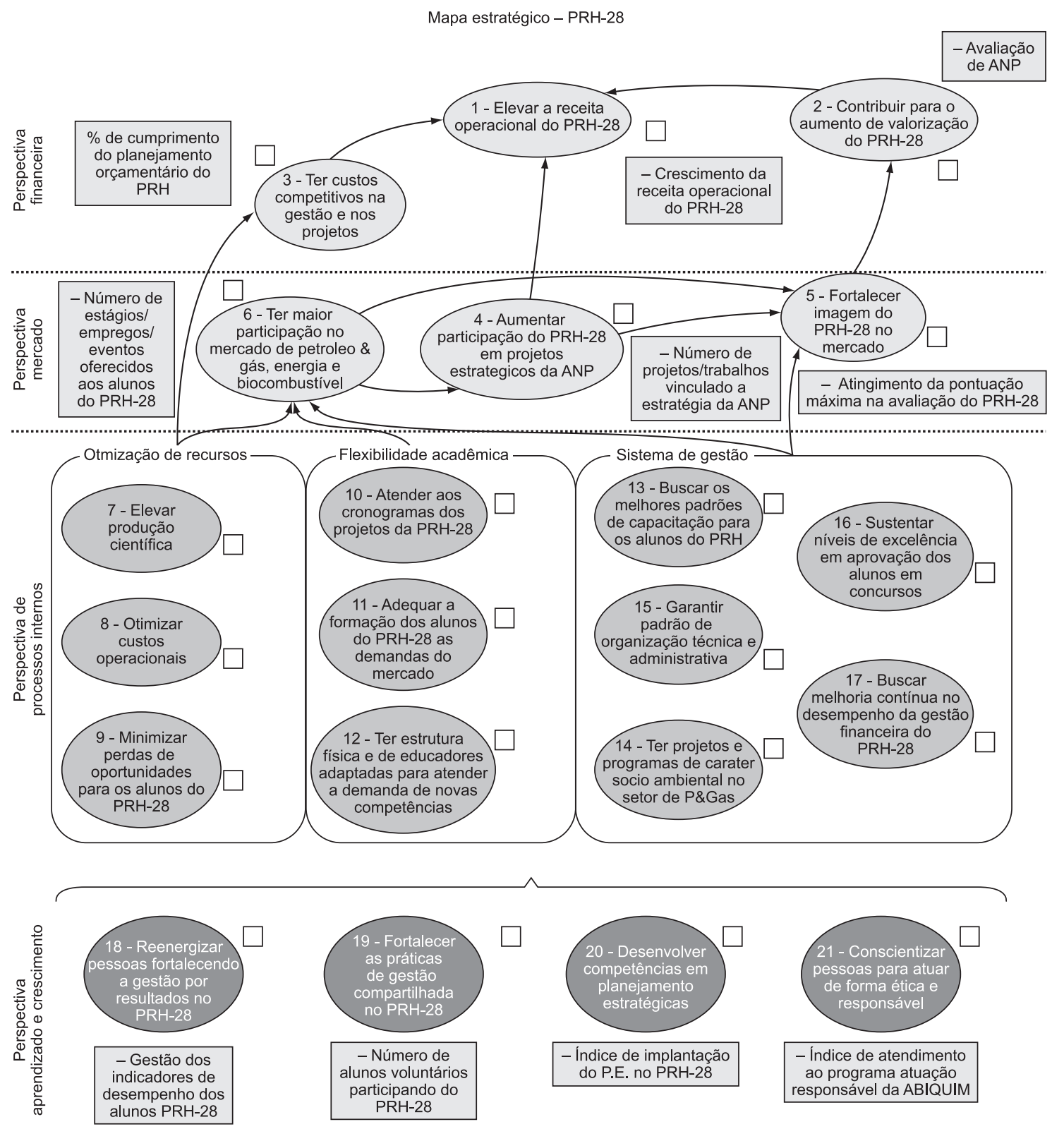

Figura 2. Mapa Estratégico montado para o PRH sob estudo.

\subsection{Método de edução bayesiano baseado em algoritmo binário}

Como já dito na subseção 2.2, a escassez de dados se torna um empecilho para modelagens mais sofisticadas. Tal dificuldade é contornada a partir de métodos específicos de coleta de opiniões, conhecidos como métodos de edução e coleta de opiniões (ECO).

Os métodos ECO podem ser classificados em diversas categorias a depender da estratégia de edução. Dentre os diversos métodos ECO, o de Firmino et al. (2008) chama a atenção por coletar opiniões do especialista sob o ensejo de equilibrar acurácia, completeza e eficiência nas suas respostas. Tal método é baseado em algoritmos de busca computacional, mais precisamente, o algoritmo de busca binária em que o especialista escolhe dentre duas metades de um intervalo e em seguida exprime seu nível de credibilidade quando da escolha. O intervalo escolhido é então particionado e o especialista é mais uma vez convidado a escolher e atribuir o nível de credibilidade quando da nova escolha. Tal algoritmo é repetido até que o especialista não se sinta mais confortável em continuar respondendo. A cada par de respostas (decisão e credibilidade) tem-se em mãos um intervalo de crença (ou Bayesiano), permitindo 
Tabela 1. Parte do plano de ação. Ações para alguns dos objetivos direcionados ao PRH de interesse.

\begin{tabular}{|c|c|c|c|c|c|c|c|}
\hline Item & Causa & Descrição & $\begin{array}{c}\text { Ações corretivas e } \\
\text { preventivas }\end{array}$ & Resp. & Prior. & Sit & Prazo \\
\hline 19.1 & $\begin{array}{l}\text { Baixo relacionamento } \\
\text { entre os alunos do } \\
\text { PRH e voluntários }\end{array}$ & $\begin{array}{l}\text { Falta de uma rede de } \\
\text { relacionamento entre } \\
\text { os alunos do PRH-28, } \\
\text { novos e antigos leva } \\
\text { a uma estagnação do } \\
\text { programa }\end{array}$ & $\begin{array}{l}\text { Criar um cadastro } \\
\text { ativo e uma rede de } \\
\text { relacionamento entre } \\
\text { os alunos do PRH }\end{array}$ & PV & & & $30 / 3 / 2008$ \\
\hline 20.1 & $\begin{array}{l}\text { Falta de participação } \\
\text { de alunos voluntários } \\
\text { ao programa PRH-28 }\end{array}$ & $\begin{array}{l}\text { Estimular a } \\
\text { participação voluntária } \\
\text { de alunos da } \\
\text { graduação ao PRH }\end{array}$ & $\begin{array}{l}\text { Alterar o critério de } \\
\text { seleção dos bolsistas } \\
\text { do PRH, incluir item } \\
\text { de aluno voluntário ao } \\
\text { programa }\end{array}$ & PV & & & $30 / 11 / 2008$ \\
\hline \multirow[t]{3}{*}{21.1} & $\begin{array}{l}\text { Falta de divulgação } \\
\text { do código de atuação } \\
\text { responsável }\end{array}$ & $\begin{array}{l}\text { Garantir que os alunos } \\
\text { do PRH ou voluntários } \\
\text { receberão treinamento } \\
\text { sobre o programa de } \\
\text { atuação responsável da } \\
\text { ABIQUIM }\end{array}$ & $\begin{array}{l}\text { Criar curso sobre o } \\
\text { programa atuação } \\
\text { responsável na } \\
\text { indústria de } \mathrm{P \& G}\end{array}$ & PV & & & $30 / 10 / 2008$ \\
\hline & & & $\begin{array}{l}\text { Criar parceria com a } \\
\text { ABIQUIM }\end{array}$ & PV & & & $30 / 6 / 2008$ \\
\hline & & & $\begin{array}{l}\text { Criar calendário de } \\
\text { palestras dentro e fora } \\
\text { da universidade }\end{array}$ & PV & & & $30 / 2 / 2008$ \\
\hline
\end{tabular}

- Em que intervalo você acha que se encontra o parâmetro de interesse?

- E com que credibilidade você faz essa afirmação?
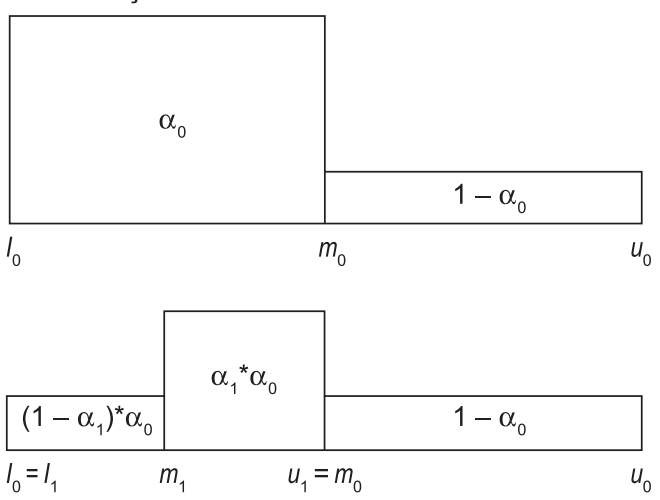

Figura 3. Ilustração do método de edução utilizado para coleta da opinião dos especialistas. Esse processo se repete até o especialista não poder distinguir mais entre os subintervalos.

estimar a distribuição de probabilidades subjacente às incertezas do especialista sobre o parâmetro em questão.

Os questionamentos envolvidos no problema em específico foram sempre direcionados à probabilidade de ocorrência de cada evento de interesse. Nesse contexto, o espaço de possibilidades é o intervalo $[0,100 \%]$.

A título de ilustração, imagine-se que o especialista seja convidado a opinar sobre a probabilidade de se executar uma ação com sucesso. Questiona-se inicialmente sobre em qual subintervalo ele acha que se encontra a probabilidade de sucesso na execução da ação, se entre $0 \%$ (limite inferior - 10) e 50\% (ponto médio - m0) ou entre 50 e 100\% (limite superior $-\mathrm{u} 0$ ). Logo em seguida, questiona-se sobre sua credibilidade, isto é, o quão certo ele está da decisão previamente tomada. Retornando às questões de decisão, caso ele tenha optado pelo primeiro subintervalo $(0,50 \%)$, o segundo subintervalo é descartado para as próximas perguntas e o primeiro subintervalo é dividido em outros dois, $0 \%$ (limite inferior $-11=10)$ a $25 \%$ (limite mediano $-\mathrm{m} 1$ ) e 25 a $50 \%$ (limite superior $-\mathrm{u} 1=\mathrm{m} 0$ ), e as perguntas se dão da mesma maneira, recursivamente. De maneira a elaborar neste processo uma distribuição de probabilidades (cuja massa probabilística deve somar um), a probabilidade de o verdadeiro valor do parâmetro se encontrar no atual intervalo equivale ao produto entre a probabilidade associada ao intervalo escolhido anteriormente e a credibilidade atribuída ao subintervalo atual. Esse processo se repete até o momento que o especialista não seja mais capaz de 
distinguir os subintervalos envolvidos. O método está ilustrado na Figura 3.

Em resumo, existem dois tipos de questões direcionadas ao especialista: questões de decisão e questões de credibilidade ou incerteza. No primeiro conjunto de questões, o especialista é questionado a escolher entre duas possíveis decisões em relação ao intervalo de interesse. No segundo conjunto, o especialista deve atribuir um nível de credibilidade à escolha tomada anteriormente. $\mathrm{O}$ processo termina pelas condições já citadas acima.

Em posse da ferramenta gerencial BSC, com o conhecimento sobre a metodologia de redes Bayesianas e do método ECO Bayesiano, Oliveira (2007) propõe um modelo em que uma RB é alimentada pelas informações do BSC traçado para o PRH-28 que possui uma gama de informações dentre as quais muitas coletadas pelo método ECO descrito nessa Subseção.

\subsection{Uso de Redes Bayesianas com o balanced scorecard}

Com o modelo teoricamente pronto, Oliveira (2007) converte a estratégia do BSC para o PRH em uma $\mathrm{RB}$, com os principais objetivos e seus respectivos planos de ação. Ferreira et al. (2008) apresentam tal conversão para uma das quatro perspectivas da análise feita em cima do modelo BSC-RB do PRH, possibilitando posteriores análises de prognóstico para os objetivos e planos de ação. A representação do mapa estratégico em forma de RB pode ser vista na Figura 4 a seguir.

Observando-se a Figura 2 e a confrontando com a Figura 4, a principal diferença é apenas ilustrativa, ou seja, a representação da $\mathrm{RB}$ em relação ao mapa estratégico não muda nenhuma relação de causa-efeito ou objetivo definido. Diferenças são percebidas apenas pela adição de alguns nós para facilitar o cálculo de probabilidades realizado pela RB.

Existe ainda outra diferença entre a representação completa do BSC por uma RB. Essa diferença se dá pela adição de outros nós que representam a influência das ações em seus respectivos objetivos. Em termos de BSC, essa influência é realizada por uma planilha paralela de orientação aos gestores, mas em RB essa influência é representada por arcos ligando nós adicionais representantes das ações. A representação completa da RB, contendo todos os objetivos e os planos de ação, está presente no Anexo I.

Após a realização da equivalência entre o mapa estratégico e a RB, os gestores do PRH foram eduzidos com o objetivo de colher deles suas incertezas acerca da probabilidade de ocorrência de cada evento relevante associado ao alcance dos objetivos. Para tanto, o método ECO apresentado em Firmino et al. (2008) e em Lins et al. (2007) é adotado. Trata-se de um método de edução indireta, no qual as respostas são manipuladas matematicamente a fim de levarem às incertezas do especialista acerca do parâmetro sob estudo.

Com base nas respostas do especialista ao método ECO adotado, é construída a distribuição acerca das suas incertezas sobre o parâmetro de interesse. Uma medida de localização, tal como a moda ou a mediana dessa distribuição, foi utilizada como resultado a ser inserido na RB.

$\mathrm{O}$ resultado desse processo é então inserido na $\mathrm{RB}$, possibilitando o cálculo das probabilidades de sucesso de cada objetivo e ação por meio de um pacote computacional desenvolvido pelos autores. Ao final desse processo, uma série de análises de prognóstico é realizada para que se infira sobre quais objetivos

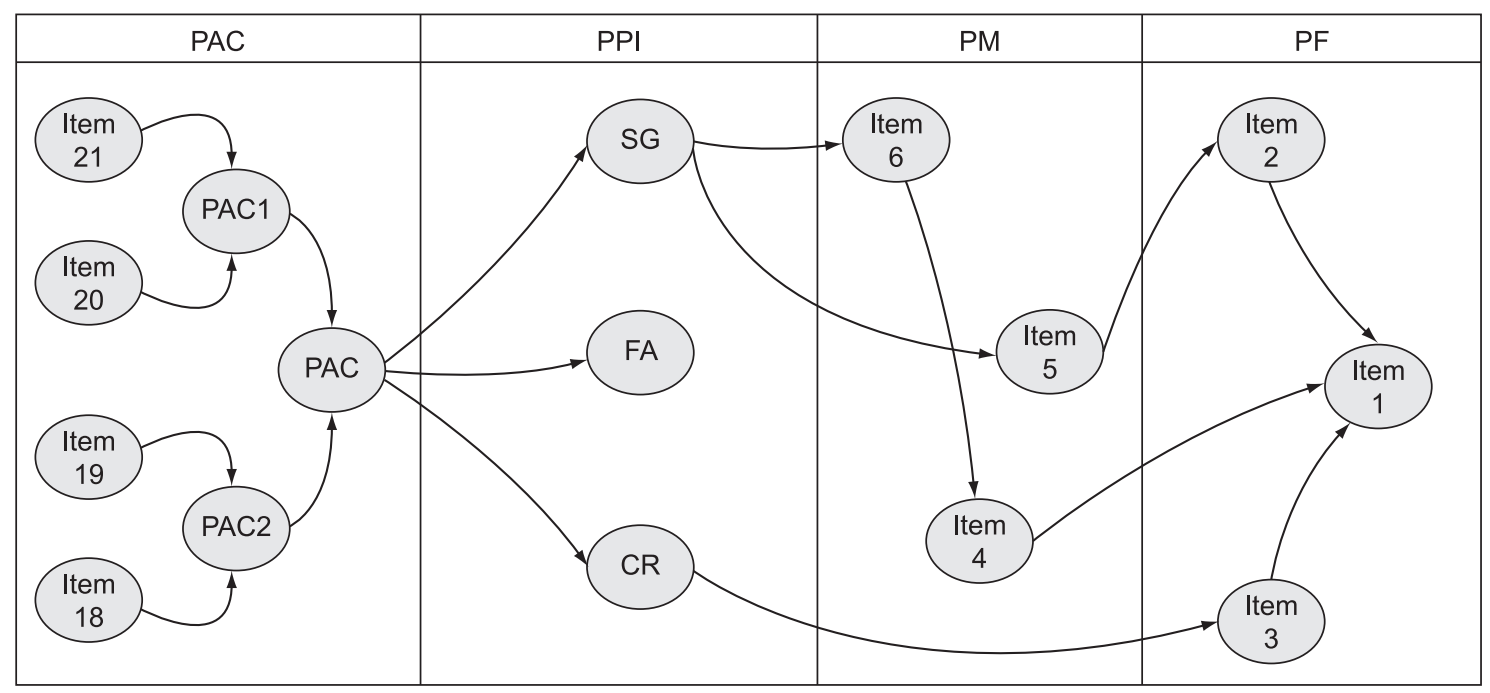

Figura 4. Representação do mapa estratégico em forma de uma Rede Bayesiana do PRH sob estudo. 
têm maior probabilidade de serem alcançados e quais são os menos prováveis.

Todos esses dados são de grande importância como comparação com os resultados reais obtidos após o ano corrente de execução da estratégia pretendida pelo modelo BSC-RB. Dessa forma, se tem como analisar o desempenho de redes Bayesianas em relação à estratégia.

\section{Resultados observados versus prognósticos do modelo}

Visto que a gama de resultados a serem explorados é imensa, o foco deste trabalho é realizar uma análise de desempenho do modelo aplicado sobre o caso de estudo do PRH confrontando os resultados observados com seus respectivos prognósticos realizados anteriormente.

A estratégia do PRH tinha como objetivo principal saber se seria possível alcançar determinada receita operacional proveniente da ANP, fazendo com que a estratégia tenha sido construída ao redor de esforços para a pontuação máxima na avaliação da ANP.

Devido à longa extensão de nomes dados aos objetivos, opta-se pela criação de códigos para cada objetivo presente na RB. A Tabela 2 mostra como se deu tal codificação.
Dado o entendimento de cada código, tem-se que a Tabela 3 mostra os prognósticos a partir da RB sobre o alcance de cada objetivo, dada a opinião dos especialistas - os gestores do PRH.

A Tabela 3 mostra que a maioria quase que absoluta dos objetivos teve mais que $50 \%$ de probabilidade de ser alcançada de acordo com as opiniões coletadas dos gestores a partir do método de edução adotado. É de se destacar que o Item 7 (sobre a produção científica dos alunos) seria pouco provável de acordo com a meta traçada. No mais, os objetivos restantes tinham uma probabilidade de sucesso moderada.

Após a realização da estratégia durante o ano de 2008, ao final da execução, realizou-se um levantamento de fracassos e sucessos alcançados pela estratégia usada. Tais resultados foram obtidos de acordo com os registros do PRH que foram fornecidos pelos gestores de maneira a confrontá-los com os resultados previstos presentes na Tabela 2. A Tabela 3 mostra os resultados obtidos e é importante ressaltar que tais resultados foram afirmados sucesso ou fracasso de acordo com os indicadores obtidos e confrontados com as metas traçadas para o ano de 2008.

Com a Tabela 4 fica possível confrontar os resultados esperados a partir da RB delineada com os resultados

Tabela 2. Codificação dos objetivos presentes no modelo BSC-RB do PRH sob estudo.

\begin{tabular}{|c|c|}
\hline Objetivo & Descrição do objetivo \\
\hline Item 1 - Maior receita & Elevar a receita operacional do PRH \\
\hline Item 2 - Valorização PRH & Contribuir para aumentar a valorização do PRH \\
\hline Item 3 - Custos competitivos & Ter custos competitivos na gestão de projetos com os alunos \\
\hline Item 4 - Maior participação ANP & Aumentar a participação do $\mathrm{PRH}$ em projetos estratégicos da ANP \\
\hline Item 5 - Imagem forte & Fortalecer a imagem do PRH no mercado \\
\hline Item 6 - Maior participação mercado & Ter maior participação no mercado de P\&G e biocombustível \\
\hline Item 7 - Mais produção científica & Aumentar a produção científica \\
\hline Item 8 - Custos ótimos & Otimizar custos operacionais \\
\hline Item 9 - Perdas mínimas para os alunos & Minimizar perdas de oportunidade para os alunos do PRH \\
\hline Item 10 - Melhoria em projetos & Buscar melhoria na gestão por projetos no $\mathrm{PRH}$ \\
\hline Item 11 - Formação adequada alunos & $\begin{array}{l}\text { Adequar a formação dos alunos do PRH às atuais demandas do } \\
\text { mercado de trabalho }\end{array}$ \\
\hline $\begin{array}{l}\text { Item } 12 \text { - Atender competências e } \\
\text { demanda }\end{array}$ & $\begin{array}{l}\text { Ter capacidade de atender a novas competências e demandas de } \\
\text { mercado }\end{array}$ \\
\hline Item 13 - Padrão de capacitação & Buscar melhores padrões de capacitação para os alunos do PRH \\
\hline Item 14 - Programas sócio-ambientais & $\begin{array}{l}\text { Ter projetos e programas de caráter sócio-ambiental no setor de } \mathrm{P} \& \mathrm{G} \text { e } \\
\text { biocombustíveis }\end{array}$ \\
\hline Item 15 - Gestão administrativa & Garantir boas práticas de gestão administrativa no PRH \\
\hline Item 16 - Níveis de excelência & Sustentar níveis de excelência dos alunos em concursos \\
\hline Item 17 - Melhoria na gestão financeira & Buscar melhoria contínua no desempenho da gestão financeiro do PRH \\
\hline Item 18 - Gestão por resultados & Reenergizar pessoas fortalecendo a gestão por resultados \\
\hline Item 19 - Gestão compartilhada do PRH & Fortalecer as práticas de gestão compartilhada no PRH \\
\hline Item 20 - Planejamento estratégico & Desenvolver competências em planejamento estratégico \\
\hline Item 21 - Conscientização de pessoas & Conscientizar as pessoas de forma ética e responsável. \\
\hline
\end{tabular}


Tabela 3. Opiniões coletadas e combinadas dos gestores do PRH em relação ao sucesso de cada objetivo.

\begin{tabular}{lc}
\hline \multicolumn{1}{c}{ Objetivo } & $\begin{array}{c}\text { Probabilidade } \\
\text { de sucesso }\end{array}$ \\
\hline Item 1 - Maior receita & $65,98 \%$ \\
Item 2 - Valorização PRH & $59,84 \%$ \\
Item 3 - Custos competitivos & $71,83 \%$ \\
Item 4 - Maior participação ANP & $64,44 \%$ \\
Item 5 - Imagem forte & $61,07 \%$ \\
Item 6 - Maior participação mercado & $53,61 \%$ \\
Item 7 - Mais produção científica & $42,27 \%$ \\
Item 8 - Custos ótimos & $61,91 \%$ \\
Item 9 - Perdas mínimas para os & $77,43 \%$ \\
alunos & $56,63 \%$ \\
Item 10 - Melhoria em projetos & $69,37 \%$ \\
Item 11 - Formação adequada alunos & $62,90 \%$ \\
Item 12 - Atender competências e & $51,90 \%$ \\
demanda & $57,94 \%$ \\
Item 13 - Padrão de capacitação & $71,24 \%$ \\
Item 14 - Programas sócio- & $62,10 \%$ \\
ambientais & $68,40 \%$ \\
Item 15 - Gestão administrativa & $60,47 \%$ \\
Item 16 - Níveis de excelência & $69,10 \%$ \\
Item 17 - Melhoria na gestão & $86,51 \%$ \\
financeira & $82,22 \%$ \\
Item 18 - Gestão por resultados & \\
Item 19 - Gestão compartilhada do & \\
PRH & Item 20 - Planejamento estratégico \\
Item 21 - Conscientização de pessoas & - \\
\hline
\end{tabular}

observados e, em seguida, traçar conclusões sobre a estratégia que o modelo ajudou a montar.

Coincidindo com as estimativas provindas do modelo, o Item 7 - produção científica - não obteve sucesso em alcançar a meta traçada. Isso pode ser explicado pelo fato de que a meta não poderia ser reajustada para se ter uma probabilidade razoável de cumpri-la, ao mesmo tempo que serviu de estímulo para que a produção científica fosse impulsionada dentro do PRH.

Os objetivos em sua maioria obtiveram sucesso no alcance das metas, mostrando coerência com o que fora traçado anteriormente pelos gestores com o auxílio do modelo BSC-RB. Mais especificamente, apenas 4 objetivos foram fracassos na estratégia, sendo que um deles já era esperado de acordo com os prognósticos do modelo. Isso mostra que os gestores tinham confiança na capacidade de se atingir as metas traçadas e alegaram que quando isso não aconteceu, ou as metas foram ajustadas ou os objetivos foram repensados, tornando o sucesso o mais viável possível para cada objetivo. Comente-se a importância das
Tabela 4. Resultados alcançados durante a execução da estratégia do PRH no ano de 2008.

\begin{tabular}{|c|c|}
\hline Objetivo & $\begin{array}{l}\text { Resultados } \\
\text { alcançados }\end{array}$ \\
\hline Item 1 - Maior receita & Sucesso \\
\hline Item 2 - Valorização PRH & Sucesso \\
\hline Item 3 - Custos competitivos & Sucesso \\
\hline Item 4 - Maior participação ANP & Sucesso \\
\hline Item 5 - Imagem forte & Sucesso \\
\hline Item 6 - Maior participação mercado & Fracasso \\
\hline Item 7 - Mais produção científica & Fracasso \\
\hline Item 8 - Custos ótimos & Sucesso \\
\hline Item 9 - Perdas mínimas para os alunos & Sucesso \\
\hline Item 10 - Melhoria em projetos & Sucesso \\
\hline Item 11 - Formação adequada alunos & Sucesso \\
\hline $\begin{array}{l}\text { Item } 12 \text { - Atender competências e } \\
\text { demanda }\end{array}$ & Sucesso \\
\hline Item 13 - Padrão de capacitação & Sucesso \\
\hline Item 14 - Programas sócio-ambientais & Sucesso \\
\hline Item 15 - Gestão administrativa & Fracasso \\
\hline Item 16 - Níveis de excelência & Sucesso \\
\hline Item 17 - Melhoria na gestão financeira & Fracasso \\
\hline Item 18 - Gestão por resultados & Sucesso \\
\hline $\begin{array}{l}\text { Item } 19 \text { - Gestão compartilhada do } \\
\text { PRH }\end{array}$ & Sucesso \\
\hline Item 20 - Planejamento estratégico & Sucesso \\
\hline Item 21 - Conscientização de pessoas & Fracasso \\
\hline
\end{tabular}

simulações neste sentido em que as estratégias podiam ser melhoradas a partir das baixas probabilidades de sucesso provenientes do modelo.

As surpresas surgiram com os fracassos dos Itens 21, 17,15 e 6 . O Item 21 e o Item 15 apresentavam altas chances de sucesso de acordo com a opinião dos gestores, o que acabou não acontecendo no final da execução do planejamento estratégico. Os gestores explicam que, apesar de tais itens terem metas ambiciosas, eram atingíveis, mas em contrapartida não dependiam apenas de seus esforços isolados. Fatores externos tais como a participação extensiva dos alunos ou ultrapassar barreiras burocráticas serviram de empecilho para que tais objetivos não fossem alcançados. Uma possível solução seria o ajuste da meta para menos, mas, caso os fatores externos não se realizassem, a meta não seria cumprida da mesma forma.

Conclusões semelhantes podem ser traçadas para o Item 6 e o Item 17. No caso do Item 6, o mercado surge como fator externo que não contribuiu para o alcance da meta, enquanto que no Item 17, os impasses burocráticos - tanto internos quanto externos - foram os obstáculos para o sucesso de tal item. 
Apesar da presença de fracasso durante o percurso da estratégia, o principal objetivo do PRH para o ano de 2008 foi alcançado com sucesso. De acordo com os resultados simulados pelo modelo BSC-RB, se esperava que, com aproximadamente $66 \%$ de certeza se alcançasse o sucesso da meta final, e o sucesso realmente foi alcançado ao final.

Além dessas análises, diagnósticos podem ser feitos pelas evidências observadas. Um diagnóstico inicial é saber qual evento mais teria influenciado na ocorrência do objetivo final em termos probabilísticos. De acordo com as simulações, o Item 20 - planejamento estratégico - apresenta a maior probabilidade de ter causado a evidência fornecida: $86,15 \%$. É interessante saber também qual seria o evento com menor poder causal sobre a ocorrência do objetivo final. As simulações indicam que o Item 7 - produção científica - possui $43,4 \%$ de ter levado ao alcance do objetivo final. Tais observações indicam que as metas traçadas para o Item 20 são mais suscetíveis ao cumprimento, enquanto que a produção científica possui metas mais difíceis de serem alcançadas.

Um diagnóstico interessante é realizado quando se tem como evidência a não ocorrência de alguns objetivos e verifica-se o que acontece com as ações direcionadas a cada objetivo. A não ocorrência do Item 7 tem como seu maior causador a elevação de produção científica, a qual apresentou uma probabilidade de não ocorrência de $84,96 \%$. Destaque-se que a criação de oportunidades apresenta baixas probabilidades de não ocorrência - 11,35\%. Para os gestores, isso mostra que mesmo com a criação de oportunidades no campo científico, os resultados não surgem por parte dos alunos.

As surpresas nos itens tidos como não alcançados anteriormente também se tornam surpresa pelo resultado das análises de diagnóstico. O Item 6 - maior participação no mercado -, tendo a não ocorrência evidenciada, apresenta ações que dificilmente não seriam executadas. Isso reforça a opinião dos gestores de que quando se trata de influência no mercado, fatores externos inevitavelmente atuam nos objetivos relacionados.

O Item 15 - gestão administrativa -, também não alcançado, apresenta um cenário semelhante após a simulação. Ações tomadas pela administração do programa em estudo dificilmente não seriam executadas, mas isso não tornou o objetivo um sucesso. Impasses burocráticos explicados pelos gestores podem ser a chave do problema nesse caso.

O Item 17 - melhoria na gestão financeira -, mesmo não tendo ocorrido, apresenta ações que não se realizam com uma probabilidade moderada. A dificuldade de realização dessas ações aliadas com impasses burocráticos são as explicações dadas pelos gestores. Observa-se que, após as simulações, os gestores agora admitem a dificuldade também na execução das ações antes não citadas como impasse.

O Item 21 - conscientização de pessoas -, quando evidenciado como não ocorrido, apresenta, como em casos anteriores, que as ações de responsabilidade dos gestores são difíceis de não serem executadas, enquanto que as ações dependentes de alunos facilmente não ocorrerão.

Isso indica que o trabalho realizado em relação à coleta das opiniões dos gestores se mostra de grande importância, pois permite aos gestores trabalhar as possibilidades de sucesso de forma fiel as incertezas que rondam os parâmetros de interesses que nesse caso são muitos. Todo esse trabalho leva os gestores a repensarem suas opiniões associando a devida incerteza a cada uma delas. Consequentemente as relações entre os objetivos e ações, os indicadores de desempenho e as metas traçadas são repensadas de maneira criteriosa para que o sucesso do objetivo final tenha a maior probabilidade de ocorrência possível.

\section{Conclusões}

O presente trabalho busca demonstrar como o modelo BSC-RB proposto por Oliveira (2007) e discutido por Ferreira et al. (2008) se comporta quando confrontado com os resultados observados em dado caso real - o Programa de Recursos Humanos da Agência Nacional do Petróleo -, obtidos após o término da execução da estratégia pretendida.

Acredita-se que um dos principais fatores para o bom desempenho do método reside no método de edução e coleta de opiniões adotado. Tal método quantifica as incertezas dos especialistas sobre dado parâmetro de interesse de maneira indireta, buscando transmitir ao modelo estimativas confiáveis dos parâmetros.

Observa-se uma coerência entre os resultados esperados e os resultados observados, sendo que os próprios gestores se deram ao trabalho de explicar os fatores que levaram aos fracassos e sucessos dos objetivos. Isso deixa claro que os gestores têm a consciência de que algumas medidas preventivas poderiam ser tomadas antes mesmo de se colocar o planejamento estratégico em ação, mas que se encontravam com limitações que os impediam de tais mudanças.

As análises de diagnóstico apresentadas após a evidência de alguns fracassos e sucessos demonstram qual conjunto de ações foi mais eficiente ou ineficiente ao se tentar cumprir cada objetivo. Com essas análises, os gestores são capazes de observar se, além de fatores externos ou fora de seu alcance, as ações pretendidas para atingir o objetivo são de alcance possível ou se alguma ou algumas delas devem ser repensadas para se aumentarem suas chances de sucesso. As análises de diagnóstico, portanto servem como mais 
uma fonte de feedback para os gestores em relação à estratégia traçada.

Sendo assim, ao final da análise verifica-se que o modelo demonstra um desempenho satisfatório ao que os gestores esperavam que acontecesse com o alcance das metas, além de criar um senso de análise das relações de causa e efeito nos gestores. Ou seja, o modelo minimiza o uso de estratégias emergenciais e fornece um conhecimento prévio de como se comportará a estratégia traçada pelos gestores. $\mathrm{O}$ mais importante é que se prognosticou o sucesso do objetivo final, o que aconteceu de fato com os resultados observados. Isso pode ser associado a como o trabalho de coleta das opiniões dos gestores fora feito. Os gestores trabalham suas opiniões e as incertezas acerca delas com mais fidelidade, abrindo assim caminhos para uma análise criteriosa do papel de cada objetivo, ação, indicador de desempenho e meta traçada dentro da estratégia.

Como limitação, a qual está ligada com a escassez de dados para a quantificação da Rede Bayesiana elaborada, os objetivos só podiam ter dois resultados: sucesso ou fracasso. Mas o fato de não se ter alcançado uma meta por completo, pode não representar um fracasso do objetivo e sim, como exemplo, um "cumprimento parcial" do objetivo. Dessa forma, uma maneira de se explorar com mais detalhes a riqueza de informações fornecida pelos objetivos, os gestores podem adicionar outros níveis de avaliação para cada objetivo, conscientes de que isso implicará em um maior trabalho de cálculos realizados pela $\mathrm{RB}$ correspondente. Além disso, se torna possível a realização de uma série de análises de diagnóstico para o conhecimento das causas que levaram ao sucesso ou ao fracasso de alguns objetivos.

Ainda, o modelo proposto mostra-se apto não apenas ao problema especificado nesse trabalho, mas para quaisquer problemas de gestão estratégica no qual se use o BSC ou outras ferramentas gerencias que possam ser modeladas por RBs. Neste sentido, a própria interação entre os diversos objetivos e metas delineados pelo BSC seria medida e assim melhor articulada.

\section{Agradecimentos}

À equipe de gestores do Programa de Recursos Humanos em estudo por cooperar tanto no fornecimento de resultados como também na modelagem inicial do modelo proposto.

\section{Referências}

AGÊNCIA NACIONAL DO PETRÓLEO, GÁS NATURAL E BIOCOMBUSTÍVEIS - ANP. Programa de Recursos Humanos. Brasília, 2009.Disponível em: <http://www. anp.gov.br/site/extras/prh/home.htm>. Acesso em: março de 2009.

AKKERMANS, H. A.; OORSCHOT, K. E. Relevance assumed: a case study of balanced scorecard development using system dynamics. Journal of the Operational Research Society, v. 56, n. 8, p. 931-941, 2005.

BLUMENBERG, S. A.; HINZ, D. J. Enhancing the Prognostic Power of IT Balanced Scorecards with Bayesian Belief Networks. In: HAWAII INTERNATIONAL CONFERENCE ON SYSTEM SCIENCES, 39., 2006, Koloua. Proceedings...

FERREIRA, R. J. et al. Modelagem das estratégias através do Balanced Scorecard e Redes Bayesianas. In: ENCONTRO NACIONAL DE ENGENHARIA DE PRODUÇÃO, 28., 2008, Rio de Janeiro. Anais...

FIRMINO, P. R. A.; SALES FILHO, R. L. M.; DROGUETT, E. L. An expert opinion elicitation method based on bayesian intervals estimation and computational algorithm: an application to oil refinery risk analysis. In: INTERNATIONAL PROBABILISTIC SAFETY ASSESSMENT AND MANAGEMENT CONFERENCE, 9., 2008, Hong Kong. Proceedings...

HERRERO, E. Balanced scorecard e a gestão estratégica: uma abordagem prática. Rio de Janeiro: Elsevier, 2005

KAPLAN, R. S.; NORTON, D. P. Estratégia em ação: balanced scorecard. Rio de Janeiro: Campus, 1997.

KAPLAN, R. S.; NORTON, D. P. Organização orientada para a estratégia. Rio de Janeiro: Campus, 2000

KORB, K. B.; NICHOLSON, A. E. Bayesian artificial intelligence. Florida: Chapman \& Hall, 2003.

LINS, I. et al. Evaluation of the efficiency of systematic elicitation methods. In: SIMPÓSIO BRASILEIRO DE PESQUISA OPERACIONAL, 39., 2007, Fortaleza. Anais...

NORREKLIT, H. The balanced on the balanced scorecard - a critical analysis of some of its assumptions. Management Accounting Research, v. 11, n. 1, p. 65-88, 2000.

OLIVEIRA, P. E. L. Aplicação de redes bayesianas na administração estratégica das organizações. Recife: Universidade Federal de Pernambuco, 2007.

OTHMAN, R. Balanced scorecard and causal model development: preliminary findings. Management Decision, v. 44, n. 5, p. 690-702, 2006.

PEARL, J. Causality: models, reasoning and inference. Los Angeles: Hardback, 2000. 400 p. 


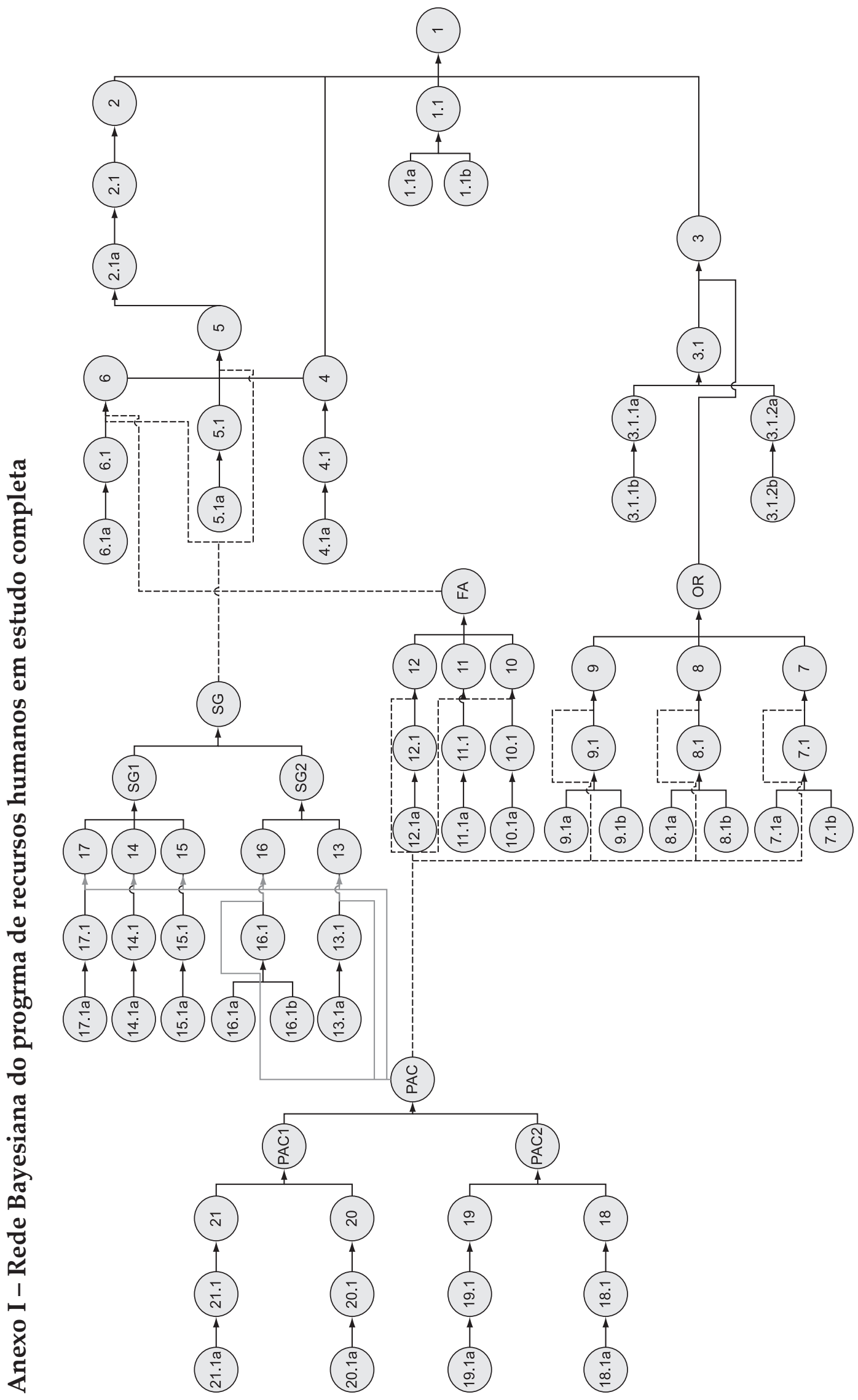

